\title{
Abstract: Multispectral Imaging Enables Visualization of Spreading Depolarizations in Gyrencephalic Brain
}

\author{
Leonardo Ayala ${ }^{1,2,3}$, SJ Wirkert ${ }^{1}$, MA Herrera ${ }^{3}$, Adrián Hernández-Aguilera ${ }^{3}$, \\ AS Vermuri ${ }^{1}$, E Santos $^{3}$, L Maier-Hein $^{1,2}$ \\ ${ }^{1}$ Division of Computer Assisted Medical Interventions, German Cancer Research \\ Center, Heidelberg, Germany. \\ ${ }^{2}$ Medical Faculty, University of Heidelberg, Heidelberg, Germany. \\ ${ }^{3}$ Department of Neurosurgery, Heidelberg University Hospital, Heidelberg, Germany. \\ 1.menjivar@dkfz-heidelberg.de
}

Spreading Depolarization (SD) is a phenomenon in the brain related to the abrupt depolarization of neurons in gray matter which results from a break-down of ion gradients across the neuron membrane and propagates like a wave of ischemia. While modulating the hemodynamic response of the SDs is a therapeutic target, the lack of imaging methods that allow for monitoring SDs with high spatiotemporal resolution hinder progress in the field. In this work, we address this bottleneck with a new method for brain imaging based on multispectral imaging (MSI). Our approach to visualizing SDs uses a machine learning-based algorithm for estimation of tissue oxygenation using MSI data acquired from the brain. Due to the lack of a gold standard method for measuring oxygen saturation in tissue, training a machine learning-based algorithm is not straightforward in this scenario. The proposed method tackles this problem with in silico training data, with the generated spectral reflectances covering a wide range of spectra that can be observed in vivo [1]. To validate our methodology in an initial feasibility study, we used a swine model of SD. A craniotomy exposed the parietal cortex, and SDs were induced using 2-5 $\mu \mathrm{L} \mathrm{KCl}$ drops placed in regions selected by visual inspection in the parietal cortex. SDs were continuously monitored with two ECoG recording strips that were placed on the lateral margins of the craniotomy. Our new approach to oxygenation estimation based on MSI was successfully applied to visualize the SDs in the gyrencephalic brain. As it has the potential to monitor SDs with high spatio-temporal resolution without a complex hardware setup, this tool could be used in studies of new treatment strategies and development of drugs that can target the hemodynamic response of the SDs.

\section{References}

1. Wirkert SJ, Vemuri AS, Kenngott HG, et al. Physiological parameter estimation from multispectral images unleashed. Proc MICCAI. 2017;1:134-141. 